\title{
The relationship between tumour budding, the tumour microenvironment and survival in patients with primary operable colorectal cancer
}

\author{
Hester C van Wyk ${ }^{*}, 1$, James H Park ${ }^{1}$, Joanne Edwards ${ }^{2}$, Paul G Horgan ${ }^{1}$, Donald C McMillan ${ }^{1}$ \\ and James J Going ${ }^{3}$ \\ ${ }^{1}$ Academic Unit of Surgery, College of Medical, Veterinary and Life of Sciences, University of Glasgow, Royal Infirmary, Glasgow \\ G31 2ER, UK; ${ }^{2}$ Unit of Experimental Therapeutics, Institute of Cancer Sciences, College of Medical, Veterinary and Life Sciences, \\ University of Glasgow, Glasgow, UK and ${ }^{3}$ University Department of Pathology, College of Medical, Veterinary and Life of Sciences, \\ University of Glasgow, Southern General Hospital, Glasgow, UK
}

Background: Tumour budding has been reported to reflect invasiveness, metastasis and unfavourable prognosis in colorectal cancer. The aim of the study was to examine the relationship between tumour budding and clinicopathological characteristics, tumour microenvironment and survival in patients with primary operable colorectal cancer.

Methods: A total of 303 patients from a prospective data set of patients with primary operable colorectal cancer were included in the study. The presence of budding was determined through assessment of all tumour-containing H\&E slides and the number of tumour buds was counted using a 10 high-powered field method. Routine pathologic sections were used to assess: tumour necrosis, the tumour inflammatory cell infiltrate using Klintrup-Makinen (KM) grade and tumour stroma percentage (TSP) combined as the Glasgow Microenvironment Score (GMS).

Results: High-grade tumour budding was present in $39 \%$ of all tumours and in $28 \%$ of node-negative tumours respectively. Highgrade budding was significantly associated with T stage $(P<0.001)$, N stage $(P<0.001)$, TNM stage $(P<0.001)$, serosal involvement $(P<0.001)$, venous invasion $(P<0.005)$, KM grade $(P=0.022)$, high tumour stroma $(P<0.001)$ and $\mathrm{GMS}(P<0.001)$. Tumour budding was associated with reduced cancer-specific survival (CSS) ( $H R=4.03 ; 95 \%$ confidence interval $(C \mathrm{C}), 2.50-6.52 ; P<0.001)$, independent of age $(\mathrm{HR}=1.47 ; 95 \% \mathrm{Cl}, 1.13-1.90 ; P=0.004)$, TNM stage $(\mathrm{HR}=1.52 ; 95 \% \mathrm{Cl}, 1.02-2.25 ; P=0.040)$, venous invasion $(\mathrm{HR}=1.73 ; 95 \% \mathrm{Cl}, 1.13-2.64 ; P=0.012)$ and $\mathrm{GMS}(\mathrm{HR}=1.54 ; 95 \% \mathrm{Cl}, 1.15-2.07 ; P=0.004)$.

Conclusions: The presence of tumour budding was associated with elements of the tumour microenvironment and was an independent adverse prognostic factor in patients with primary operable colorectal cancer. Specifically high tumour budding stratifies effectively the prognostic value of tumour stage, venous invasion and GMS. Taken together, tumour budding should be assessed routinely in patients with primary operable colorectal cancer.

Colorectal cancer is a leading cause of cancer-related deaths worldwide with up to $50 \%$ of patients dying of recurrent disease within 5 years (Hyslop and Waldman, 2013). Although the TNM staging system forms the basis of pathological staging in patients with colorectal cancer, additional prognostic markers are required to improve the prediction of likely outcome and therefore the management of patients.

*Correspondence: Dr HC van Wyk; E-mail: h.van-wyk.1@research.gla.ac.uk

Received 13 January 2016; revised 9 May 2016; accepted 13 May 2016; published online 14 June 2016

(c) 2016 Cancer Research UK. All rights reserved 0007-0920/16 
Over the last decade, tumour budding has gained momentum as a possible marker of aggressive tumour behaviour and therefore a supplement to the current TNM staging system, classified as an 'additional' prognostic factor in colorectal cancer by the American Joint Committee on Cancer and International Union against Cancer (AJCC/UICC) (Compton, 2006).

Tumour budding has been defined as the presence of isolated single cells or small clusters of cells composed of up to five cells (Ueno et al, 2002). Tumour budding is proposed to reflect the process of epithelial-mesenchymal transition (EMT) whereby highly differentiated epithelial mucosal cells develop to invasive phenotypes (Zlobec and Lugli, 2010). Tumour budding appears to be associated with adverse clinicopathological characteristics and poor outcome and most studies report tumour budding as a prognostic factor independent of other tumour features (van Wyk et al, 2015).

To date, the tumour microenvironment has mainly been described as a host immune reaction represented by periand intratumoural immune cell infiltrates (Roxburgh and McMillan, 2012) and the amount of stroma in the tumour (Mesker et al, 2007).

These have recently combined in a tumour microenvironment score, the Glasgow Microenvironment Score (GMS) (Park et al, 2015).

The aim of the present study was examine the relationship between tumour budding, tumour microenvironment and survival in patients with primary operable colorectal cancer.

\section{PATIENTS AND METHODS}

Patients. From a prospective database of patients with colorectal cancer who underwent potentially curative resection between 1997 and 2008 in a single surgical unit at the Glasgow Royal Infirmary, 303 patients were included in the study. Patients who had metastatic disease at diagnosis or underwent neoadjuvant therapy and patients who died within 30 days of surgery were excluded. Tumours were staged according to the fifth edition of the AJCC/UICC-TNM staging system. Local ethical committee approval was granted.

\section{Histopathology}

Venous invasion. Only extramural vascular invasion was recorded. Venous invasion (VI) was assessed routinely with elastica since 2003. Before 2003, VI was retrospectively stained with elastica (Roxburgh et al, 2010).

Budding. The H\&E slides containing the highest number of budding foci were selected. Tumour sections were scanned using a Hamamatsu Nano Zoomer (Welwyn Garden City, UK) at $\times 20$ magnification, and visualisation was carried out using the Slide path Digital Image Hub, version 4.0.1 (Slide path, Leica Bio Systems, Milton Keynes, UK). For quantification, the areas with the highest density of peritumoural budding was selected under scanning magnification and the number of tumour buds (tumour cells with up to five nuclei or single tumour cells) were counted in 10 high-power fields (10 HPFs), as first described by Horcic et al (2013) and Karamitopoulou et al (2013). Size of field (1 HPF per 1 grid) was $50 \mu \mathrm{m}$ (Figure $1 \mathrm{~A}$ ). The total number of buds was used for analysis. To ensure reliability, co-scoring of 25 randomly selected cases (10HPFs) was carried out by HCvW and consultant pathologist JJG. The interobserver intraclass correlation coefficient (ICCC) for the scores was $0.766(P<0.001)$. The remaining slides were scored by $\mathrm{HCvW}$ and these results were used in the analysis.

Tumour necrosis. Tumour necrosis was graded semiquantitatively as 'absent' (none), 'focal' ( $<10 \%$ of tumour area), 'moderate'
(10-30\%) or 'extensive' ( $>30 \%)$ as previously described (Richards et al, 2012).

Klintrup-Makinen grade. Klintrup-Makinen (KM) grade was used to assess the generalised inflammatory infiltrate semiquantitatively. The H\&E-stained sections of the deepest point of tumour invasion were used, whereby inflammatory cell density at the invasive margin was graded using a four-point scale and subsequently classified as low grade (no increase or mild/patchy increase in inflammatory cells) or high grade (prominent inflammatory reaction forming a band at the invasive margin, or florid cup-like infiltrate at the invasive edge with destruction of cancer cell islands) as previously described (Klintrup et al, 2005; Roxburgh et al, 2009).

Tumour stroma percentage. Tumour stroma percentage (TSP) was assessed semiquantitatively using full sections of the deepest point of tumour invasion; the proportion of stroma was calculated as a percentage of the visible field, excluding areas of mucin deposition or necrosis.

Tumours were subsequently graded as low TSP (50\%) or high TSP (>50\%) as previously described (Park et al, 2014).

Glasgow Microenvironment Score. Glasgow Microenvironment Score is a cumulative prognostic score that combined KM grade and TSP and has shown to have independent prognostic value. Scores used for GMS: 0 (KM strong), 1 (KM weak/low TSP) and 2 (KM weak/high TSP) (Park et al, 2015).

$M M R$ protein expression. Expression was reported as MMR proficient (strong nuclear staining with positive immune cells) or MMR deficient (staining intensity is either weak or patchy with normal immune cell infiltrate, or negative with complete loss of expression and normal immune cell expression). Methods used were as previously described (Park et al, 2016).

Statistical analysis. An optimal cutoff score for the determination of 'high-grade' budding was performed by a receiver operating characteristic curve analysis with cancer-specific survival (CSS) as endpoint.

The $\chi^{2}$ test was used to examine associations between clinicopathological data and the presence of tumour budding. Survival curves were constructed with the Kaplan-Meier method and the influence of a given parameter on survival was assessed with the log-rank test. Multivariate survival analysis with calculation of hazard ratios (HRs) was performed using Cox's regression. A stepwise backward procedure derived a final model of variables with a significant independent relationship. Deaths up to 15 March 2013 were included in the analysis. The $P$-values of $\leqslant 0.05$ were considered as statistically significant. Statistical analysis was performed using the SPSS software version 21 (SPSS Inc., Chicago, IL, USA).

\section{RESULTS}

Patient characteristics and pathologic variables. The study comprised 303 patients with the mean patient age at the time of resection of 68 years (range, 32-98 years) with $52 \%$ of patients as males. Of the tumours, $76 \%$ occurred in the colon and $24 \%$ in the rectum.

In terms of $\mathrm{T}$ stage, tumour invaded to the submucosa in $6(2 \%)$, to muscularis propria in $22(7 \%)$, to subserosa in $188(62 \%)$ and to peritoneum in 87 (29\%) patients. No positive lymph nodes were found in 197 (65\%) patients, 1-3 lymph nodes contained tumour in $85(28 \%)$ patients, and $\geqslant 3$ lymph nodes were positive in 21 (7\%) patients. Of the tumours, $87 \%$ were well and moderately 

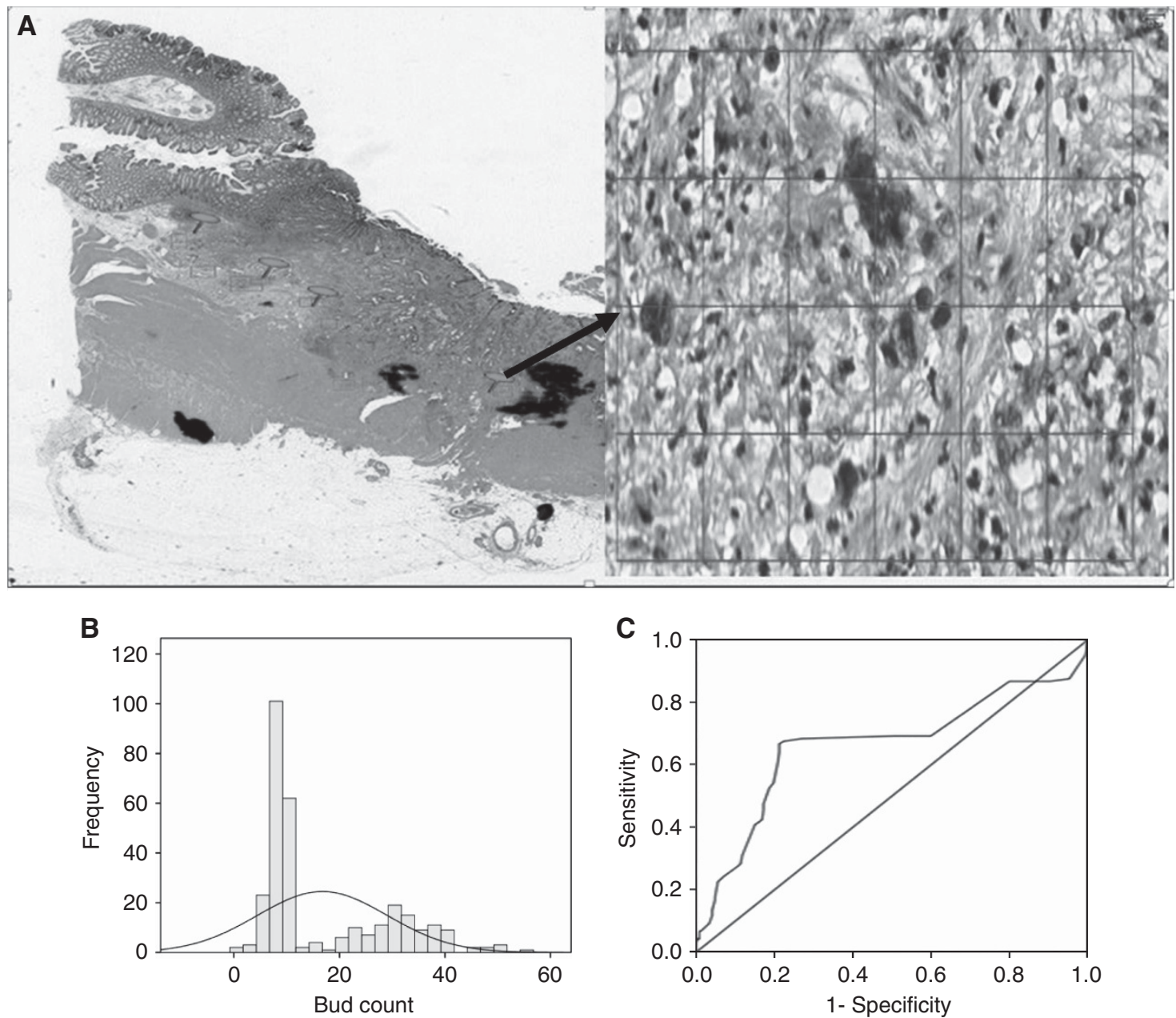

Figure 1. Assessment of tumour budding. (A) The 10 HPF method showing placement grid (1 HPF), (B) distribution of tumour buds and (C) ROC analysis.

differentiated, whereas $13 \%$ were poorly differentiated. Of the tumours, $79(26 \%)$ had serosal involvement, whereas in $22(7 \%)$ resection margins were involved. Venous invasion was present in $41 \%$ of colorectal tumours and in $35 \%$ of the 197 node-negative tumours. Of the patients, $76(25 \%)$ received adjuvant therapy and $51 \%$ of patients with TNM stage III received adjuvant chemotherapy.

Determination of optimal score for high-grade budding. The total bud count per $10 \mathrm{HPF}$ varied from 0 to 63 buds. In order to identify the threshold for the number of buds, ROC analysis was carried out. The distribution of tumour buds is shown in Figure 1B and the optimal threshold of buds was derived from the ROC analysis (Figure 1C; point of inflection $=20$ buds; $A U C=0.663$; 95\% confidence interval (CI), 0.597-0.729; $\mathrm{P}<0.001$ ).

Incidence of tumour budding and association of budding with clinicopathologic variables. The overall detection rate for highgrade budding was $39 \%$ and $28 \%$ for the whole and node-negative cohort, respectively. High-grade budding was associated with T stage $(P<0.001)$, N stage $(P<0.001)$ and TNM stage $(P<0.001)$. In terms of tumour characteristics, high-grade budding was associated with serosal involvement $(P<0.001)$, VI $(P<0.01)$, KM grade $(P<0.05)$, TSP $(P<0.001)$ and GMS $(P<0.001$; Table 1).

Of the 215 patients who had MMR status assessed, 63 out of 74 (85\%) MMR-competent patients had high tumour budding in comparison with 11 out of 74 (15\%) MMR-deficient patients $(P=0.592)$.

The relationships between clinicopathological characteristics of node-negative colorectal tumours and high grade budding are shown in Supplementary Table 1. High-grade budding was associated with $\mathrm{T}$ stage $(P<0.010)$, serosal involvement $(P<0.010)$, VI $(P<0.05)$, KM grade $(P<0.05)$, TSP $(P<0.001)$ and GMS $(P<0.001)$.

Role of tumour budding as prognostic factor. The relationships between clinicopathological characteristics of colorectal tumours and CSS are shown in Table 2. Median follow-up was 61 (26-95) months during which there were 187 deaths, 89 from cancer. On univariate analysis, age $(P<0.018)$, T stage $(P<0.001), \mathrm{N}$ stage $(P<0.001), \quad$ TNM stage $(P<0.001)$, VI $\quad(P<0.001)$, GMS $(P<0.001)$ and tumour budding $(P<0.001)$ were associated with reduced CSS.

On multivariate analysis, age $(\mathrm{HR}=1.47 ; 95 \% \mathrm{CI}, 1.13-1.90$, $P=0.004)$, TNM stage $(\mathrm{HR}=1.52 ; 95 \% \mathrm{CI}, 1.02-2.25 ; P=0.040)$, VI $(\mathrm{HR}=1.73 ; 95 \% \mathrm{CI}, 1.13-2.64 ; P=0.012)$, GMS $(\mathrm{HR}=1.54$; $95 \% \mathrm{CI}, 1.15-2.07 ; P=0.004)$ and tumour budding $(\mathrm{HR}=4.03$; 95\% CI, 2.50-6.52; $P<0.001)$ were independently associated with reduced survival.

In TNM stage II disease, low and high tumour budding was associated with 5 -year survival/s.e. of $82 \%$ (5) and $36 \%$ (8) $(P<0.001)$. In TNM stage III disease, low and high tumour budding was associated with 5 -year survival/s.e. of $71 \%$ (9) and $31 \%(7)(P<0.001$; Figure 2A and B). 
Table 1. The relationship between tumour budding and clinicopathological characteristics in patients with primary operable colorectal cancer $(n=303)$

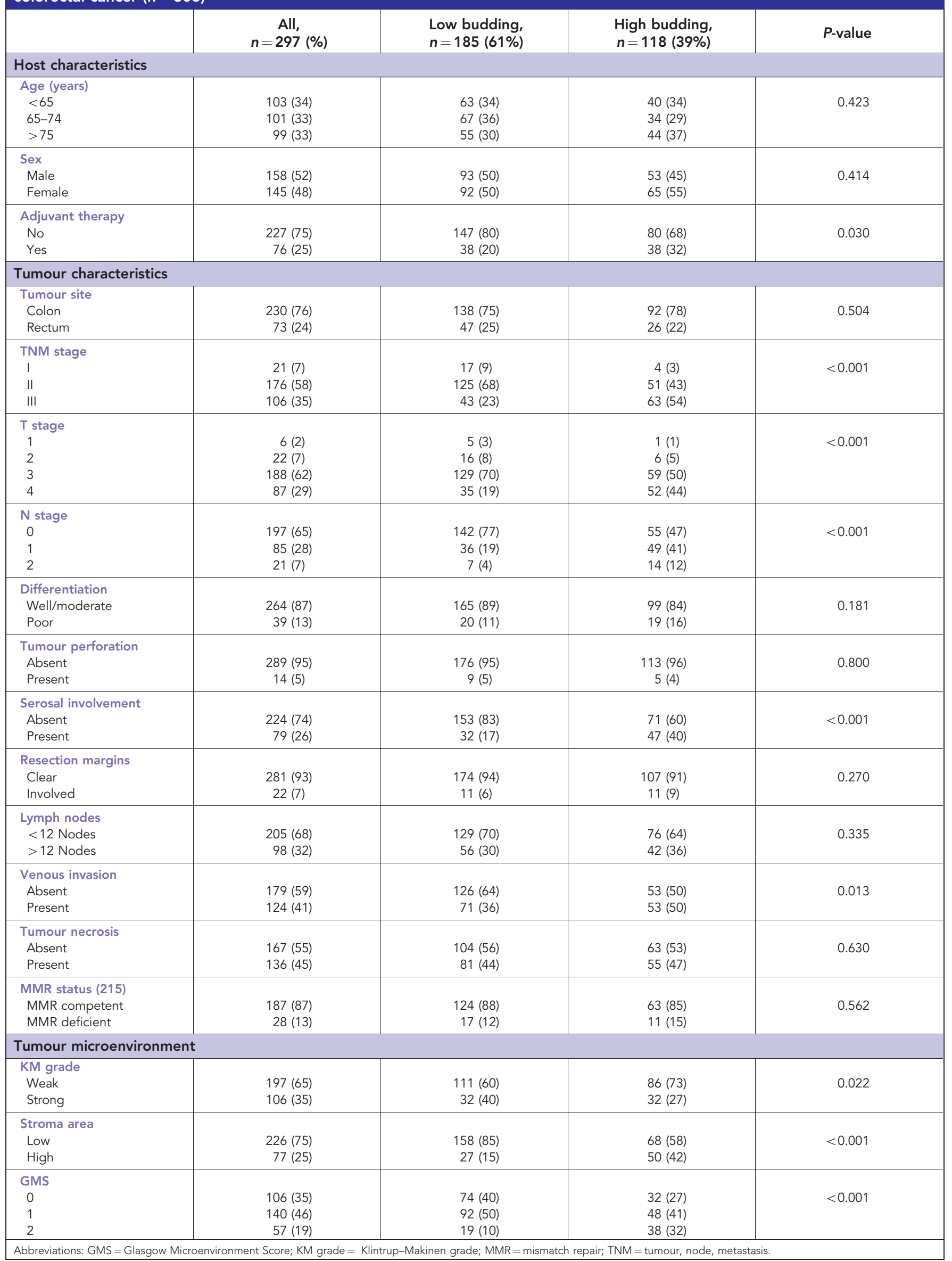


Table 2. The relationship between tumour budding, clinicopathological characteristics and cancer-specific survival in patients with primary operable colorectal cancer $(n=303)$

\begin{tabular}{|c|c|c|c|c|}
\hline & \multicolumn{2}{|c|}{ Univariate analysis } & \multicolumn{2}{|c|}{ Multivariate analysis } \\
\hline Age ( $<65 / 65-75$ years/ $>75$ years $)$ & $1.37(1.06-1.78)$ & 0.018 & $1.47(1.13-1.90)$ & 0.004 \\
\hline Site (colon/rectum) & $0.74(0.44-1.26)$ & 0.270 & & \\
\hline T stage $(1 / 2 / 3 / 4)$ & $1.98(1.38-2.85)$ & $<0.001$ & - & \\
\hline Venous invasion (absent/present) & $2.22(1.46-3.39)$ & $<0.001$ & $1.73(1.13-2.64)$ & 0.012 \\
\hline Tumour necrosis (absent/present) & $1.43(0.94-2.16)$ & 0.095 & & \\
\hline MMR status & $1.02(0.49-2.15)$ & 0.952 & & \\
\hline $\begin{array}{l}\text { Tumour microenvironment } \\
\text { GMS (0/1/2) } \\
\text { Tumour budding (low/high) }\end{array}$ & $\begin{array}{l}1.99(1.48-2.67) \\
5.97(3.73-9.56)\end{array}$ & $\begin{array}{l}<0.001 \\
<0.001\end{array}$ & $\begin{array}{l}1.54(1.15-2.07) \\
4.03(2.50-6.52)\end{array}$ & $\begin{array}{r}0.004 \\
<0.001\end{array}$ \\
\hline
\end{tabular}

In the absence of VI, low and high tumour budding was associated with 5-year survival/s.e. of $82 \%$ (5) and $46 \%$ (8) $(P<0.001)$. In the presence of VI, low and high tumour budding was associated with 5 -year survival/s.e. of $78 \%$ (7) and $23 \%(7)$ $(P<0.001$; Figure $2 \mathrm{C}$ and $\mathrm{D})$.

In $\mathrm{GMS}=0$, low and high tumour budding was associated with 5 -year survival/s.e. of $84 \%(5)$ and $44 \%(12)(P<0.001)$. In $\mathrm{GMS}=1$, low and high tumour budding was associated with 5 -year survival/s.e. of $74 \%(6)$ and $37 \%(9)(P<0.001)$. In $\mathrm{GMS}=2$, low and high tumour budding was associated with 5 -year survival/s.e. of $82 \%(9)$ and $21 \%(8)(P<0.001$; Figure 3$)$.

In the MMR-competent group, low and high tumour budding was associated with 5 -year survival/s.e. of $76 \%(5)$ and $34 \%(7)$ $(P<0.001)$. In the MMR-deficient group, low and high tumour budding was associated with 5 -year survival/s.e. of $80 \%(11)$ and 49\% (16) $(P<0.001)$. Tumour budding effectively stratified MMRcompetent status, but the numbers in the MMR-deficient group were low (Supplementary Figure 1).

\section{DISCUSSION}

The results of the present study showed that tumour budding was associated with TNM stage, serosal involvement, VI and a weaker inflammatory cell infiltrate and more stroma. Moreover, tumour budding was independently associated with CSS. Taken together, these results suggest a complex relationship between tumour budding and the tumour microenvironment and disease progression in patients with primary operable colorectal cancer.

In the present study, high-grade budding occurred in $39 \%$ of tumours and in $28 \%$ of node-negative tumours and are consistent with results from previous studies (Ueno et al, 2004; Ha et al, 2005; Kevans et al, 2011; Betge et al, 2012). Tumour budding was examined in H\&E-stained sections with the $10 \mathrm{HPF}$ method and an optimal prognostic threshold for high-grade budding was successfully implemented that yielded a cutoff (15-20 buds), similar to previous reports (Prall et al, 2005; Wang et al, 2009; Gujam et al, 2015).

The 10 HPF method using cytokeratin-stained slides was developed and validated by Horcic et al (2013) and Karamitopoulou et al (2013). As H\&E slides are readily available, the $10 \mathrm{HPF}$ method (H\&E) can potentially contribute to the long overdue implementation of tumour budding in routine pathology practice. Although previous work showed improved detection rates of tumour budding with cytokeratin and in comparison with $\mathrm{H} \& \mathrm{E}$, the interobserver agreements are reported to be superior with cytokeratin stains (Koelzer et al, 2015; Kai et al, 2016), and such immunohistochemistry appears not to improve the prognostic value (Suzuki et al, 2009; Puppa et al, 2012; van Wyk et al, 2015). Therefore, where the number of tumour buds identified is below a defined threshold, cytokeratin may be useful to assist in the assessment of difficult cases and training to introduce tumour budding into routine pathology practice. This would be similar to the RCP recommendation for routine clinical pathology assessment of VI.

In the present study tumour budding and the GMS were shown to have independent prognostic value. There are a number of potential difficulties in incorporating these measures into routine clinical pathological practice. However, with tumour budding and the GMS being measured in routine H\&E slides, the additional complexity is minimised. Moreover, it offers the possibility of computerised assessment in the same slide that may minimise the complexity and subjectivity of assessment. For example, an automated assessment algorithm for the $\mathrm{KM}$ score in routine $\mathrm{H} \& \mathrm{E}$ slides has been reported (Forrest et al, 2014). A similar approach could be applied to tumour stroma and to tumour budding in routine H\&E slides.

Recently, Dawson et al (2014) proposed that in patients with colorectal cancer, high-grade tumour budding was associated with aggressive molecular and biological features. Budding tumour cells have been proposed to undergo an EMT like event, losing expression of epithelial differentiation markers while gaining the capacity to express mesenchymal and stemness markers (Brabletz et al, 2005). Grigore et al (2016) suggested that most EMT processes in tumour buds are not complete and that tumour buds undergo partial EMT, with at least a subset of tumours displaying a true hybrid, single-cell E/M phenotype in their buds. Nevertheless, the EMT can be triggered by a diverse set of stimuli including growth factor signalling, tumour-stromal cell interactions and hypoxia. According to Righi et al (2015), tumour budding is associated with hypoxia induced by hypovascularisation at the advancing front of colorectal cancer. Budding cells are proposed to escape such hypoxia by expressing a HIF-1a-mediated hypoxic tumour phenotype that increases their potential to bud and disseminate, perhaps through VI.

In addition, it would appear that tumour budding is able to degrade the peritumoural connective tissue, evading the host's 

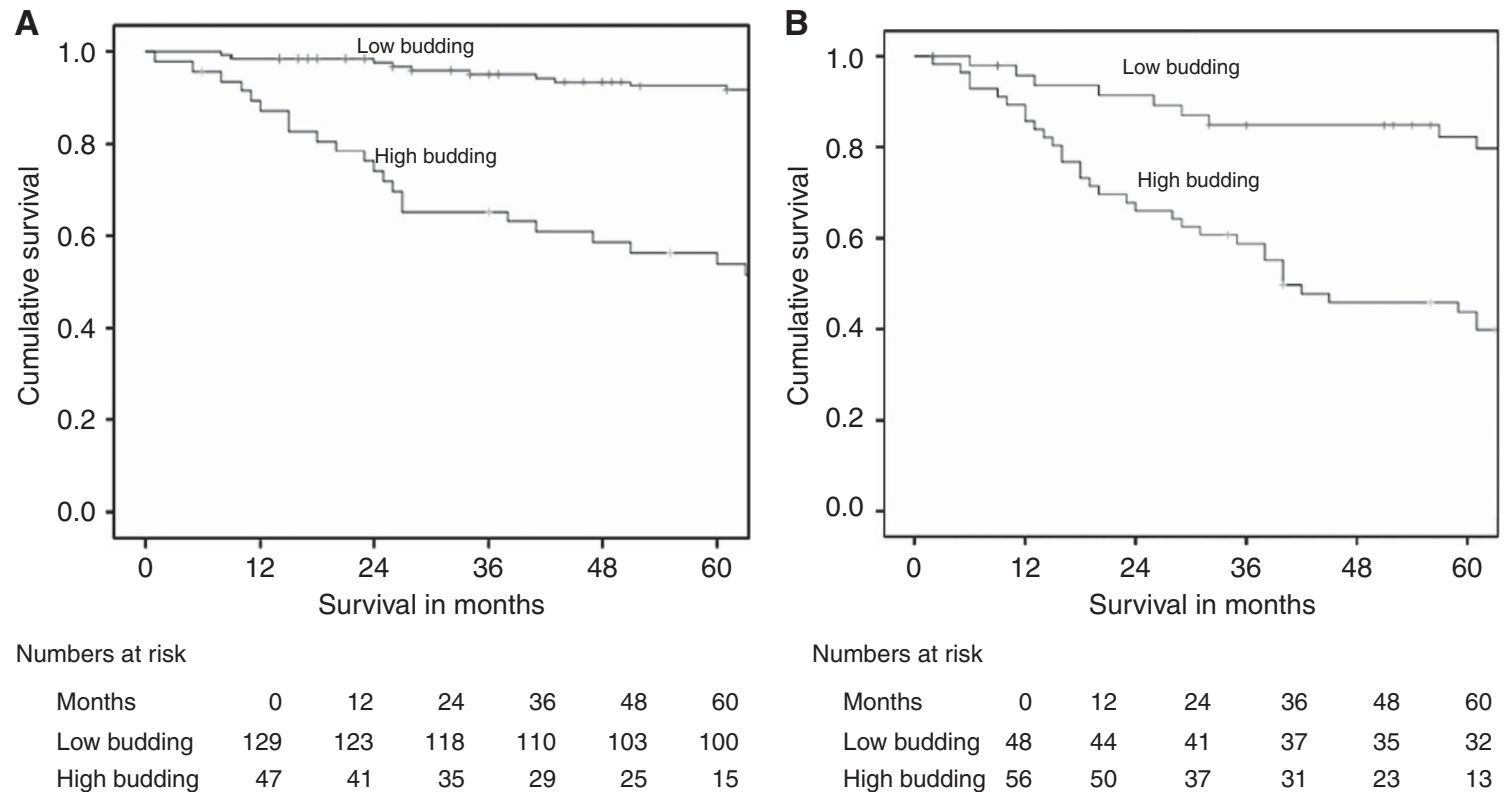

\section{Numbers at risk}

$\begin{array}{lrrrrrr}\text { Months } & 0 & 12 & 24 & 36 & 48 & 60 \\ \text { Low budding } & 48 & 44 & 41 & 37 & 35 & 32 \\ \text { High budding } & 56 & 50 & 37 & 31 & 23 & 13\end{array}$
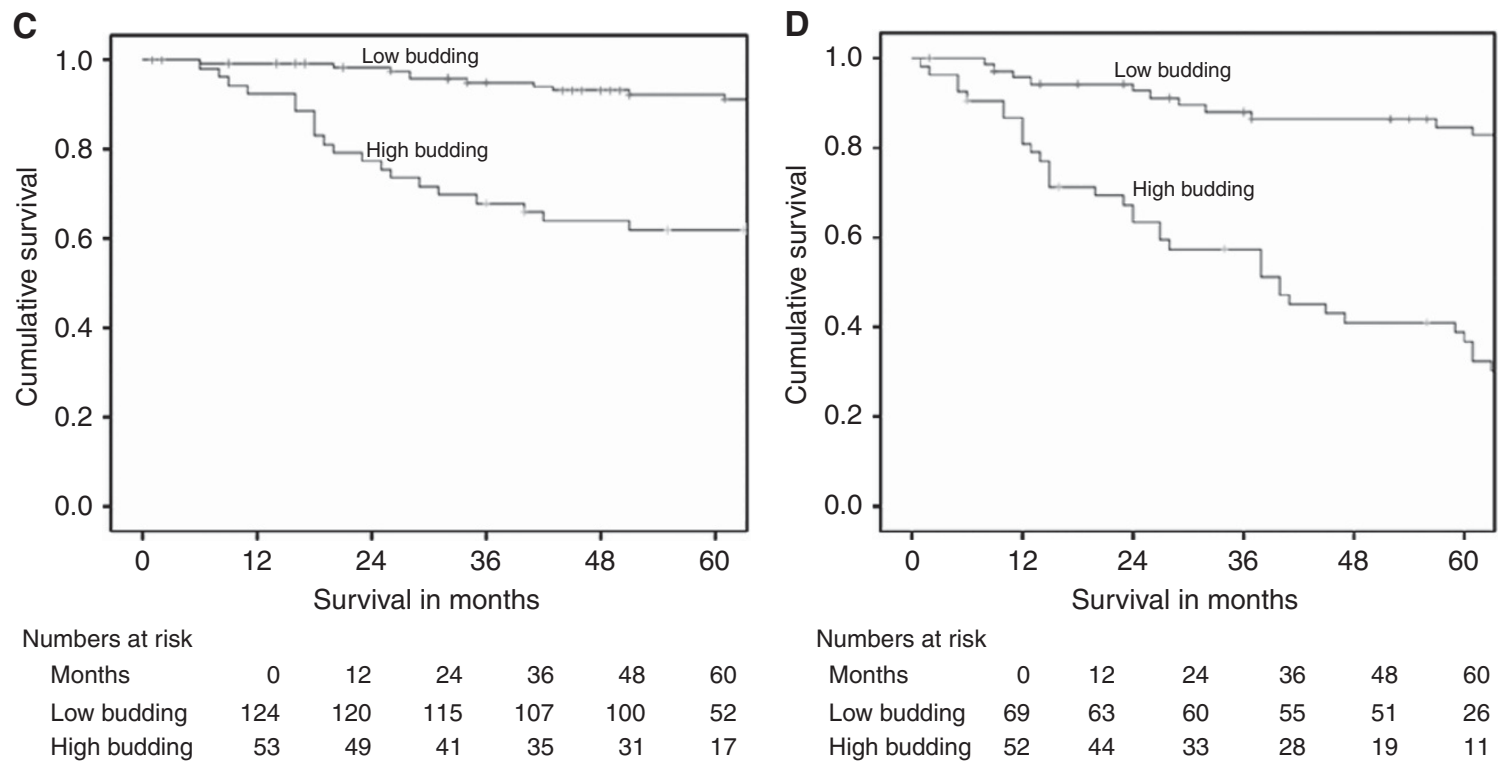

Figure 2. Relationship between budding and clinicopathological factors. (A and B) Relationship between high- and low-grade tumour budding and cancer-specific survival in patients with TNM stage II and III primary operable colorectal cancer $(P<0.001)$. (C and $\mathbf{D})$ Relationship between tumour budding, venous invasion and cancer-specific survival in patients with primary operable colorectal cancer $(P<0.001)$.

response and infiltrate lymphatic and blood vessels leading to local and distant metastasis (Lugli et al, 2012; Koelzer et al, 2015). Indeed, in the present study tumour budding was significantly associated with more tumour stroma and a weaker inflammatory cell infiltrate. These results might suggest that stromal elements interact through signalling molecules that potentially drive tumour budding and cancer progression (Hill et al, 2005).

Of particular interest is the association between tumour budding and tumour microenvironment as it is not known whether tumour budding is a result of the tumour microenvironment or whether it causes changes in the tumour microenvironment. Although highgrade tumour budding and the characteristics of the tumour microenvironment (i.e., stromal infiltration and loss of the conspicuous inflammatory cell infiltrate) may represent differing end points of common precursor genetic pathways, one may occur as a consequence of the other. Indeed, it was of interest that the proportion of patients with high-grade tumour budding increased significantly with increasing GMS (GMS0: 30\% vs GMS2: 36\%).
These results may suggest that tumour budding is a result of changes in the tumour microenvironment rather than the cause.

As both tumour budding and the GMS were independently associated with poor outcome, tumour budding was combined with GMS as follows: high KM and low stroma percentage and low budding $=0$, low $\mathrm{KM}=1$, low $\mathrm{KM}$ and high stroma percentage $=2$, low $\mathrm{KM}$ and high stroma percentage and high budding $=3$. The combined GMS and tumour budding effectively stratifies survival in patients with primary operable colorectal cancer $(\mathrm{HR}=2.16$; 95\% CI, 1.65-2.82; $P<0.001$; Figure 4 ).

The limitations of the present study were as follows: the study was conducted at a single centre and therefore the results need to be further validated before any definite change of practice can be recommended and with reference to the pathogenetic relationship between tumour budding and microenvironment, the selection of $\mathrm{H} \& \mathrm{E}$-stained sections of the deepest point of tumour invasion for assessing inflammatory infiltrate and tumour stroma percentage, as well as H\&E slides containing the highest number of budding foci 


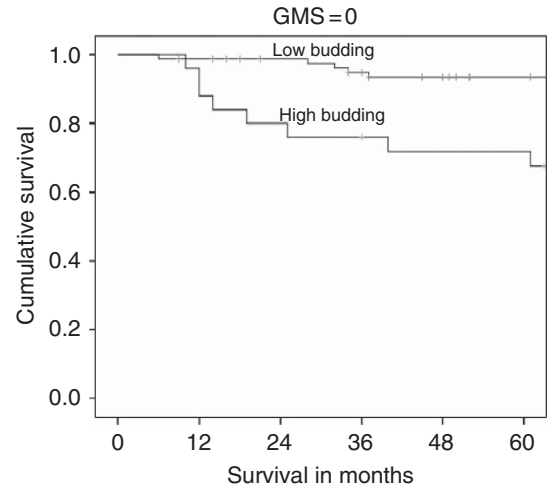

Number at risk

$\begin{array}{lllllll}\text { Months } & 0 & 12 & 24 & 36 & 48 & 60 \\ \text { Low budding } & 80 & 76 & 73 & 68 & 63 & 32 \\ \text { High budding } & 25 & 24 & 20 & 18 & 17 & 11\end{array}$

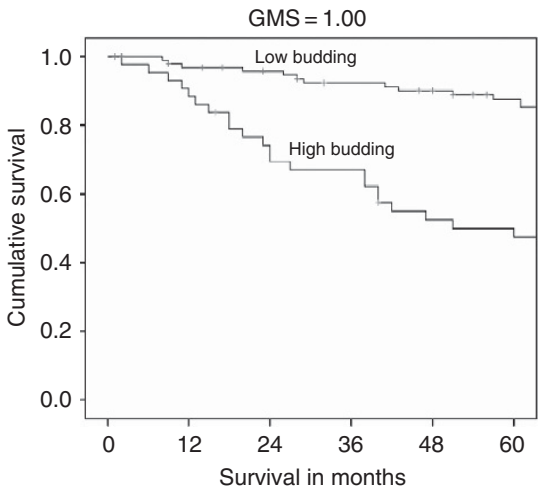

Number at risk

$\begin{array}{lllllll}\text { Months } & 0 & 12 & 24 & 36 & 48 & 60 \\ \text { Low budding } & 95 & 88 & 85 & 80 & 76 & 39\end{array}$

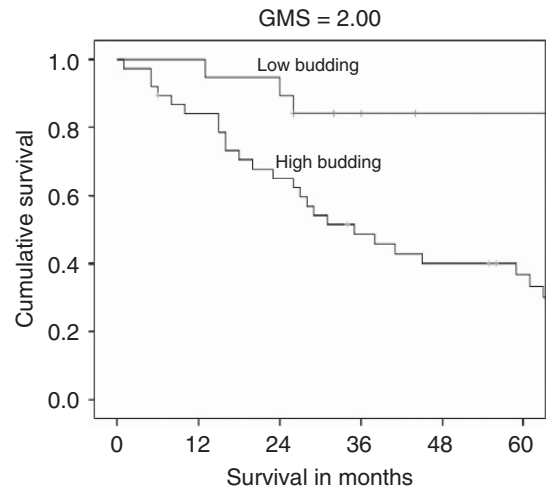

Number at risk

$\begin{array}{lllllll}\text { Months } & 0 & 12 & 24 & 36 & 48 & 60 \\ \text { VI absent } & 19 & 19 & 17 & 13 & 12 & 6 \\ \text { VI present } & 37 & 31 & 23 & 17 & 13 & 7\end{array}$

Figure 3. Relationship between tumour budding, Glasgow Prognostic Score (GMS) and cancer-specific survival in patients with primary operable colorectal cancer $(P<0.001)$.

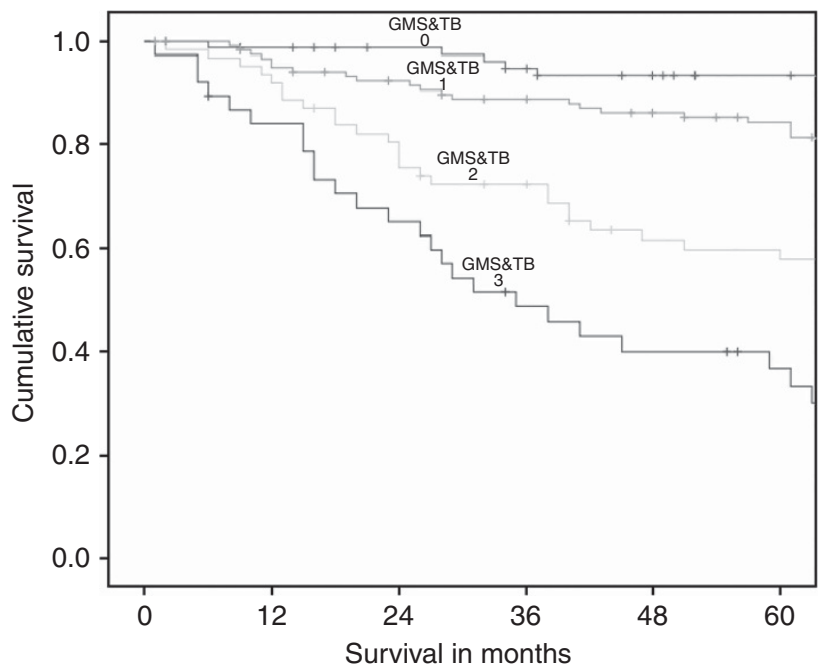

Figure 4. Relationship between combined score of Glasgow Prognostic Score and tumour budding and cancer-specific survival in patients with primary operable colorectal cancer $(P<0.001)$.

for tumour budding assessment that may act as confounding factors for the interpretation of the results. Therefore, assessment of the intratumour distance between the selected areas may be useful to include in the analysis in future studies.

\section{CONCLUSION}

The assessment of tumour budding with $\mathrm{H} \& \mathrm{E}$ is reliable and effectively risk stratifies patients with primary operable colorectal cancer. Tumour budding can potentially be integrated into existing staging systems and should be considered for implementation in routine clinical practice. The results also suggest that the presence of tumour budding is determined with the tumour microenvironment and this relationship is worthy of further investigation.

\section{CONFLICT OF INTEREST}

The authors declare no conflict of interest.

\section{REFERENCES}

Betge J, Kornprat P, Pollheimer MJ, Lindtner RA, Schlemmer A, Rehak P, Vieth M, Langner C (2012) Tumor budding is an independent predictor of outcome in AJCC/UICC stage II colorectal cancer. Ann Surg Oncol 19: 3706-3712.

Brabletz T, Jung A, Spaderna S, Hlubek F, Kirchner T (2005) Migrating cancer stem cells - an integrated concept of malignant tumour progression. Nat Rev Cancer 5: 744-749.

Compton CC (2006) Key issues in reporting common cancer specimens. Arch Pathol Lab Med 130: 318-324.

Dawson H, Koelzer VH, Karamitopoulou E, Economou M, Hammer C, Muller DE, Lugli A, Zlobec I (2014) The apoptotic and proliferation rate of tumour budding cells in colorectal cancer outlines a heterogeneous population of cells with various impacts on clinical outcome. Histopathology 64: 577-584.

Forrest R, Guthrie GJ, Orange C, Horgan PG, McMillan DC, Roxburgh CS (2014) Comparison of visual and automated assessment of tumour inflammatory infiltrates in patients with colorectal cancer. Eur J Cancer 50: $544-552$.

Grigore AD, Jolly MK, Jia D, Farach-Carson MC, Levine H (2016) Tumor budding: the name is EMT. Partial EMT. J Clin Med 5: 51.

Gujam FJA, McMillan DC, Mohammed ZMA, Edwards J, Going JJ (2015) The relationship between tumour budding, the tumour microenvironment and survival in patients with invasive ductal breast cancer. $\mathrm{Br} J$ Cancer 113: $1066-1074$.

Ha SS, Choi HJ, Park KJ, Kim JM, Kim SH, Roh YH, Kwon HC, Roh MS (2005) Intensity of tumor budding as an index for the malignant potential in invasive rectal carcinoma. Cancer Res Treat 37: 177-182.

Hill R, Song Y, Cardiff RD, Van Dyke T (2005) Selective evolution of stromal mesenchyme with p53 loss in response to epithelial tumorigenesis. Cell 123: 1001-1011.

Horcic M, Koelzer VH, Karamitopoulou E, Terracciano L, Puppa G, Zlobec I, Lugli A (2013) Tumor budding score based on 10 high-power fields is a promising basis for a standardized prognostic scoring system in stage II colorectal cancer. Hum Pathol 44: 697-705.

Hyslop T, Waldman SA (2013) Molecular staging of node negative patients with colorectal cancer. J Cancer 4: 193-197.

Kai K, Aishima S, Aoki S, Takase Y, Uchihashi K, Masuda M, NishijimaMatsunobu A, Yamamoto M, Ide K, Nakayama A, Yamasaki M, Toda S (2016) Cytokeratin immunohistochemistry improves interobserver variability between unskilled pathologists in the evaluation of tumor budding in T1 colorectal cancer. Pathol Int 66: 75-82.

Karamitopoulou E, Zlobec I, Kölzer V, Kondi-Pafiti A, Patsouris ES, Gennatas K, Lugli A (2013) Proposal for a 10-high-power-fields scoring method for the assessment of tumor budding in colorectal cancer. Mod Pathol 26: 295-301. 
Kevans D, Wang LM, Sheahan K, Hyland J, O’Donoghue D, Mulcahy H, O'Sullivan J (2011) Epithelial-mesenchymal transition (EMT) protein expression in a cohort of stage II colorectal cancer patients with characterized tumor budding and mismatch repair protein status. Int J Surg Pathol 19: 751-760.

Klintrup K, Makinen JM, Kauppila S, Vare PO, Melkko J, Tuominen H, Tuppurainen K, Makela J, Karttunen TJ, Makinen MJ (2005) Inflammation and prognosis in colorectal cancer. Eur J Cancer 41: 2645-2654.

Koelzer VH, Dawson H, Andersson E, Karamitopoulou E, Masucci GV, Lugli A, Zlobec I (2015) Active immunosurveillance in the tumor microenvironment of colorectal cancer is associated with low frequency tumor budding and improved outcome. Transl Res 166: 207-217.

Koelzer VH, Zlobec I, Berger MD, Cathomas G, Dawson H, Dirschmid K, Seelentag W (2015) Tumor budding in colorectal cancer revisited: results of a multicenter interobserver study. Virchows Archiv 466: 485-493.

Lugli A, Karamitopoulou E, Zlobec I (2012) Tumour budding: a promising parameter in colorectal cancer. Br J Cancer 106: 1713-1717.

Mesker WE, Junggeburt J, Szuha K, de Heer P, Morreau H, Tanke HJ, Tollenaar RA (2007) The carcinoma-stromal ratio of colon carcinoma is an independent factor for survival compared to lymph node status and tumor stage. Anal Cell Pathol 29: 387-398.

Park JH, Powell AG, Roxburgh CS, Horgan PG, McMillan DC, Edwards J (2016) Mismatch repair status in patients with primary operable colorectal cancer: associations with the local and systemic tumour environment. Br J Cancer 114: 562-570.

Park JH, McMillan DC, Powell AG, Richards CH, Horgan PG, Edwards J, Roxburgh CS (2015) Evaluation of a tumor microenvironment-based prognostic score in primary operable colorectal cancer. Clin Cancer Res 21: $882-888$.

Park JH, Richards CH, McMillan DC, Horgan PG, Roxburgh CSD (2014) The relationship between tumour stroma percentage, the tumour microenvironment and survival in patients with primary operable colorectal cancer. Ann Oncol 25: 644-651.

Prall F, Nizze H, Barten M (2005) Tumour budding as prognostic factor in stage I/II colorectal carcinoma. Histopathology 47: 17-24.

Puppa G, Senore C, Sheahan K, Vieth M, Lugli A, Zlobec I, Pecori S, Wang LM, Langner C, Mitomi H, Nakamura T (2012) Diagnostic reproducibility of tumour budding in colorectal cancer: a multicentre, multinational study using virtual microscopy. Histopathology 61: 562-575.

Richards CH, Roxburgh CSD, Anderson JH, McKee RF, Foulis AK, Horgan PG, McMillan DC (2012) Prognostic value of tumour necrosis and host inflammatory responses in colorectal cancer. Br J Surg 99: 287-294.
Righi A, Sarotto I, Casorzo L, Cavalchini S, Frangipane E, Risio M (2015) Tumour budding is associated with hypoxia at the advancing front of colorectal cancer. Histopathology 66: 982-990.

Roxburgh CS, Salmond JM, Horgan PG, Oien KA, McMillan DC (2009) Tumour inflammatory infiltrate predicts survival following curative resection for node-negative colorectal cancer. Eur J Cancer 45 : $2138-2145$.

Roxburgh CS, McMillan DC, Anderson JH, McKee RF, Horgan PG, Foulis AK (2010) Elastica staining for venous invasion results in superior prediction of cancer-specific survival in colorectal cancer. Ann Surg 252: 989-997.

Roxburgh CSD, McMillan DC (2012) The role of the in situ local inflammatory response in predicting recurrence and survival in patients with primary operable colorectal cancer. Cancer Treat Res 38: 451-466.

Suzuki A, Togashi K, Nokubi M, Koinuma K, Miyakura Y, Horie H, Lefor AT, Yasuda Y (2009) Evaluation of venous invasion by Elastica van Gieson stain and tumor budding predicts local and distant metastases in patients with T1 stage colorectal cancer. Am J Surg Pathol 33: 1601-1607.

Ueno H, Jones AM, Wilkinson KH, Jass JR, Talbot IC (2004) Histological categorisation of fibrotic cancer stroma in advanced rectal cancer. Gut 53 : 581-586.

Ueno H, Murphy J, Jass JR, Mochizuki H, Talbot IC (2002) Tumour 'budding' as an index to estimate the potential of aggressiveness in rectal cancer. Histopathology 40: 127-132.

van Wyk HC, Park JH, Roxburgh C, Horgan P, Foulis A, McMillan DC (2015) The role of tumour budding in predicting survival in patients with primary operable colorectal cancer: a systematic review. Cancer Treat Rev 2: 151-159.

Wang LM, Kevans D, Mulcahy H, O’Sullivan J, Fennelly D, Hyland J, Diarmuid O, Sheahan K (2009) Tumor budding is a strong and reproducible prognostic marker in T3N0 colorectal cancer. Am J Surg Pathol 33: 134-141.

Zlobec I, Lugli A (2010) Epithelial mesenchymal transition and tumor budding in aggressive colorectal cancer: tumor budding as oncotarget. Oncotarget 1: 651-661.

This work is published under the standard license to publish agreement. After 12 months the work will become freely available and the license terms will switch to a Creative Commons AttributionNonCommercial-Share Alike 4.0 Unported License.

Supplementary Information accompanies this paper on British Journal of Cancer website (http://www.nature.com/bjc) 